which are made of silicon dioxide only about 1.5-nm thick (about four atomic layers of $\mathrm{SiO}_{2}$ ). Further decreases in the thickness of the $\mathrm{SiO}_{2}$ would result in unacceptably high leakage current that would drain batteries and overheat chips. To continue the advancement of microelectronics, materials with higher dielectric constant $(k)$ and higher thickness will be needed to replace the $\mathrm{SiO}_{2}$.

This is the main research focus of Roy G. Gordon and co-workers at Harvard University. In the August issue of Chemistry of Materials, the research team reported the use of chemical vapor deposition (CVD) and atomic layer deposition (ALD) techniques to deposit metal silicates and metal oxides. The researchers use reactions between the vapors of metal alkylamides and tris(tert-alkoxy)silanols. For CVD, a reaction chamber is supplied with continuous flows of the vapors of tetrakis(dimethylamino)hafnium and tris(tert-butoxy)silanol, which were dissolved separately in tetradecane solutions and then flash-vaporized. With substrate temperatures $\sim 250-350^{\circ} \mathrm{C}$, the deposited product is a hafnia-silica glass, $\mathrm{HfO}_{2}\left(\mathrm{SiO}_{2}\right)_{x}$.

For ALD, pulses of each vapor are dosed alternately and separately into the reaction chamber, producing the same composition with very uniform thickness, even inside small holes. ALD showed high growth rates of $0.3-0.4 \mathrm{~nm}$ per deposition cycle, a level higher than traditional ALD reactions. This ALD procedure can also be modified to deposit metal oxides by replacing the pulses of tris(tert-butoxyl)silanol vapor with water vapor. By varying the ratio of silanol pulses to water pulses, the ratio of silicon to hafnium can be controlled from $x \sim 0$ to 3 . Rutherford backscattering measurements showed impurity levels of nitrogen and carbon to be very low ( $<2$ at.\%). Additional attractive characteristics included relative smoothness $(<0.5 \%)$ and lack of cracks and pinholes in the thin films over domains of $300 \mathrm{~mm}$, as determined by atomic force microscopy, scanning electron microscopy, and transmission electron microscopy. No special pretreatment was needed for silicon and glassy carbon substrates.

Volatile amides are known for many metals, including $\mathrm{Li}, \mathrm{Na}, \mathrm{K}, \mathrm{Be}, \mathrm{Y}, \mathrm{Ti}, \mathrm{Zr}$, $\mathrm{Hf}, \mathrm{V}, \mathrm{Nb}, \mathrm{Ta}, \mathrm{Cr}, \mathrm{Mo}, \mathrm{W}, \mathrm{Mn}, \mathrm{Fe}, \mathrm{Co}, \mathrm{Ni}$, $\mathrm{Cu}, \mathrm{Zn}, \mathrm{Al}, \mathrm{Ga}, \mathrm{Ge}, \mathrm{Sn}, \mathrm{Pb}, \mathrm{Sb}$, and the lanthanide and actinide metals. Thus, according to the researchers, similar techniques could be used to deposit metal oxides and silicates for these metals.

In contrast to many CVD and ALD techniques, this process uses no metal chlorides, which can result in chloride impurities and possible substrate/equipment corrosion. Also, some metal chlorides, such as those of yttrium and lanthanum, are not volatile enough for use in CVD or ALD.

This technique may provide a way to continue Moore's law improvements in computing power by using high- $k$ materials to replace leaky gate insulators of silicon dioxide.

MATHEW M. MAYE

\section{Model Explains Capillary Condensation of Fluids in Disordered Mesoscopic Materials}

Using a mean-field density functional theory for a disordered lattice-gas model during their investigation of the phenomenon of capillary condensation of fluids in disordered mesoporous materials, researchers have discovered that the hysteresis loop can present itself both in the presence and the absence of a true equilibrium phase transition and that capillary criticality is not linked with the vanishing of the hysteresis loop. Furthermore, as Edouard Kierlik and co-workers from the Laboratoire de Physique Théorique des Liquides at the Université Pierre et Marie Curie and the Department of Chemical Engineering at the University of Massachussetts have reported in the July 30 issue of Physical Review Letters, the system can "jump" from one grand potential minimum to another along the adsorption and desorption isotherms such that thermodynamic behavior is erratic.

According to the researchers, their simulations indicate the presence of a large number of metastable states inside the main hysteresis loop. According to researcher Martin Luc Rosinberg of Université Pierre et Marie Curie, these metastable states are responsible for the typical history-dependent behavior of fluids adsorbed in disordered porous solids. The value of the solid-fluid interaction is crucial for determining whether an underlying equilibrium first-order phase transition actually occurs (i.e., a jump in the density of the adsorbed fluid).

The work of Kierlik and co-workers provides a means for understanding the experimentally observed hysteresis loops and temperature-dependence of sorption isotherms in capillary condensation studies. The "scanning curves" obtained by changing the sign of the chemical potential along adsorption and desorption branches agree with experimental observations, whereas previous models have not properly captured such features. The researchers said that the ability to reproduce observed physical behavior is a critical factor in determining a model's predictive capacity.
On a broader scale, this research provides an instance where theory can contribute key understanding by focusing on information that lies outside of conventional "restrictions": Often, theory studies pursue simulations designed to obtain equilibrium information and to present thermodynamically consistent results. Many of the interesting features obtained in this study specifically fall outside such a "narrow" regime.

The researchers draw a connection between their results for capillary condensation phenomena and the macroscopic avalanche of low-temperature ferromagnets. The researchers hope that the observation of such features in capillary condensation of real materials will provide additional support for the applicability of the model Hamiltonian and assumptions employed in this study. Predicting as-yet-unseen features and explaining unusual observed behavior are the ultimate goals of most materials simulations, they said.

EMILY JARVIS

\section{Silicon Nanowire Structure Displays Enhanced Optical Birefringence}

To further enhance the use of birefringent crystals for controlling the polarization state of light in optical applications, an international group of researchers from the Technical University of München, Germany, and Koba University in Japan, has demonstrated a way of realizing a silicon-based optical device based on the anisotropic dielectric nanostructuring of silicon wafers. The merit of this experiment is the realization in the laboratory of a material with higher values of the anisotropy parameter $\Delta n$ than of any other crystal found in nature.

As reported in the August 15 issue of Optics Letters, the method used to synthesize the Si wafers is anodic etching of bulk $\mathrm{Si}$ in 1:1 volumes of hydrofluoric acid and ethanol solution, resulting in a spongelike network of interconnected Si nanowires. The size of the nanowires is a function of the doping level of the substrate, the concentration of the etching agent, and the etching-current density. Transmission electron microscopy revealed that the pores resulting from etching propagate independently of the orientation of the substrate in a [100] equivalent crystallographic direction. To achieve in-plane anisotropy of the refractive index, the wafers were etched on the (110) plane, resulting in different refractive indices for light polarized along [110] and [001] directions. To determine $\Delta n=n_{[1 \overline{1} 0]}-n_{[001]}$, two methods were involved: transmission measurements in 